\title{
Recurrent pyelonephritis due to NDM-1 metallo-beta- lactamase producing Pseudomonas aeruginosa in a patient returning from Serbia, France, 2012
}

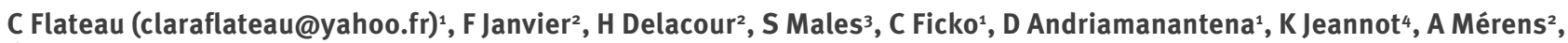
C Rapp ${ }^{1}$

1. Service des maladies infectieuses et tropicales, Hôpital d'instruction des armées Bégin, Saint-Mandé, France

2. Service de microbiologie-hygiène, Hôpital d'instruction des armées Bégin, Saint-Mandé, France

3. Service de médecine et maladies infectieuses, Hôpital Henri Duffaut, Avignon, France

4. Centre National de la Résistance aux Antibiotiques, Laboratoire de Bactériologie, Hôpital Jean Minjoz, Besançon, France

Flateau C, Janvier F, Delacour H, Males S, Ficko C, Andriamanantena D, Jeannot K, Mérens A, Rapp C. Recurrent pyelonephritis due to NDM-1 metallo-beta-lactamase producing Pseudomonas aeruginosa in a patient returning from Serbia, France, 2012. Euro Surveill. 2012;17(45): pii=20311. Available online: http://www.

eurosurveillance.org/ViewArticle.aspx?Articleld $=20311$

We describe the first isolation in France of a NewDelhi metallo-beta-lactamase-1 (NDM-1) producing Pseudomonas aeruginosa. In March 2012, a patient with history of prior hospitalisation in Serbia was diagnosed in France with acute pyelonephritis due to NDM-1 producing $P$. aeruginosa. Clinical and microbiological cure was obtained under appropriate antibiotic treatment. Two months later, she presented with a recurrence due to the same bacteria, with a favourable evolution. During both hospitalisations, contact isolation precautions were implemented and no crosstransmission was observed.

In March 2012, a patient with a history of prior hospitalisation in Serbia was diagnosed in France with acute pyelonephritis due to New Delhi metallo-beta-lactamase-1 (NDM-1) producing Pseudomonas aeruginosa.

\section{Case report}

In February 2012, a woman in her early 60s was referred to the Infectious Diseases Department of the Military Hospital Bégin (Saint-Mandé, France) for an acute pyelonephritis. She reported having undergone a surgical intervention in Serbia in November 2011. She stayed one month in hospital, with urinary catheterisation of undetermined duration but less than one month. The medical records reported a first treatment with cefuroxime and streptomycin just after surgery, and a history of fever, drowsiness and inflammatory syndrome two weeks after surgery, treated with ceftriaxone and streptomycine. Laboratory data were unavailable.

Since her return to France, in early February 2012, she complained of urinary frequency, dysuria and urinary incontinence. In late February, she presented to her general practitioner (GP) with fever $\left(38.7^{\circ} \mathrm{C}\right)$, vomiting, diarrhoea and diffuse abdominal pain and she was referred to our hospital. White blood cell count was $4,470 / \mathrm{mm}^{3}$ (norm: $4,000-10,000 / \mathrm{mm}^{3}$ ), C-reactive protein $52 \mathrm{mg} / \mathrm{L}$ (norm: $<5 \mathrm{mg} / \mathrm{L}$ ), creatinine $36 \mu \mathrm{mol} / \mathrm{L}$ (norm: 62-106 $\mu \mathrm{mol} / \mathrm{L}$ ).

The urinalysis recovered 29,106 leukocytes $/ \mathrm{mL}$ and $10^{6} \mathrm{CFU} / \mathrm{ml}$ P. aeruginosa serotype 011 (HIABP11). The rectal swab, performed for multidrug resistant bacteria screening according to the French recommendations for patients with a history of hospitalisation abroad in the previous year [1], was also positive for P. aeruginosa. Blood cultures remained negative.

Kidney ultrasonography was normal, kidney CT-scan showed a left pyelonephritis without abscess or urinary obstruction. The patient underwent urinary catheterisation and three weeks antibiotic treatment with aztreonam (2g TID) and colistin (2 million units TID).

\section{Antimicrobial sensitivity testing} and molecular diagnostics

For the isolates from urine and rectal swabs, antimicrobial drug susceptibility testing was performed by the disk diffusion method on Mueller-Hinton agar (I2A Laboratories, Perols, France) and interpreted according to the European Committee on Antimicrobial Susceptibility Testing (EUCAST) recommendations [2]. It demonstrated resistance to imipenem, meropenem, doripenem, to all antipseudomonal cephalosporins, aminoglycosides, fluoroquinolones, rifampicine and tigecycline. Both isolates remained susceptible to piperacillin-tazobactam and intermediate to aztreonam (Table 1). Minimal inhibitory concentrations (MICs) of selected antimicrobials determined by microdilution and Etest (Biomérieux, Marcy l'Etoile, France) confirmed the results and showed susceptibility to colistin. The metallo-betalactamase-production screening in the meropenem-dipicolinic acid combined disk test (Klebsiella pneumoniae carbapenemas (KPC) + metallo-beta-lactamase (MBL) Confirm ID Pack, Rosco Diagnostica, Taastrup, Denmark) and in E-test with 
TABLE 1

Antibiotic susceptibility of Pseudomonas aeruginosa serotype 011 (HIABP11) strain isolated in a patient with history of previous hospitalisation in Serbia, France 2012

\begin{tabular}{|l|c|c|}
\hline Antibiotic & MIC $(\mu \mathrm{g} / \mathrm{mL})$ & Susceptibility \\
\hline Ticarcillin & $>256$ & $\mathrm{R}$ \\
\hline Ticarcillin/Clavulanic acid & $>256$ & $\mathrm{R}$ \\
\hline Piperacillin & 12 & $\mathrm{~S}$ \\
\hline Piperacillin/Tazobactam & 12 & $\mathrm{~S}$ \\
\hline Ceftazidime & $>256$ & $\mathrm{R}$ \\
\hline Cefepime & $>256$ & $\mathrm{R}$ \\
\hline Aztreonam & 3 & $\mathrm{I}$ \\
\hline Imipeneme & $>32$ & $\mathrm{R}$ \\
\hline Meropeneme & $>32$ & $\mathrm{R}$ \\
\hline Doripenem & $>32$ & $\mathrm{R}$ \\
\hline Tobramycin & $>256$ & $\mathrm{R}$ \\
\hline Gentamicin & $>256$ & $\mathrm{R}$ \\
\hline Amikacin & 192 & $\mathrm{R}$ \\
\hline Ciprofloxacin & $>32$ & $\mathrm{~S}$ \\
\hline Colistin & 2 & $\mathrm{R}$ \\
\hline Doxycycline & & \\
\hline Tigecycline & 32 & $\mathrm{R}$ \\
\hline
\end{tabular}

MIC: minimal inhibitory concentration

I: intermediate; R: resistant; S: susceptible.

imipenem alone or combined with EDTA (Biomérieux, Marcy l'Etoile, France) was positive.

PCR for carbapenemases, including MBL bla $a_{\mathrm{IMP}}$, blaVIM, $b a_{\mathrm{NDM}}$ revealed that HIABP11 harboured $b l a_{\mathrm{NDM}}$. Sequencing showed $100 \%$ homology with $b a_{\mathrm{NDM}-1} . \mathrm{PCR}$ for detection of other beta-lactamase genes, plasmidborne quinolone resistance genes and methylases were negative.

\section{Monitoring of plasmatic and urinary concentrations of antibiotics}

Aztreonam plasmatic peak and residual concentrations were 40 times and 4 times the MIC respectively for a dosage of $2 \mathrm{~g}$ TID, while urine concentration was 166 times the MIC. Colistin plasmatic residual concentration (12 hours after injection) was under the MIC (0.7 $\mathrm{mg} / \mathrm{L}$, while the expected residual concentration is 2 $\mathrm{mg} / \mathrm{L}$, eight hours after injection); urine concentration was 10 times the MIC (Table 2).

Fever and abdominal pain resolved within 48 hours after administration of antibiotics while urinary incontinence persisted, requiring prolonged urinary catheterisation. Urinalysis 72 hours after the beginning of antibiotic treatment was normal and did not show any bacteria growth. The patient was discharged from hospital 21 days after admission.

During the stay at our hospital, a squamous-cell carcinoma of the oropharynx had been discovered and the patient underwent a first chemotherapy course at the beginning of May and was discharged from our hospital. One week later, she experienced dysuria and abdominal pain, without fever or flank pain. She was treated with ofloxacin (10 days) and prednisolone (seven days) by her GP. The urinalysis showed $42 \times 10^{4}$ leukocytes $/ \mathrm{mL}$ (significant leukocytes count $>10^{4} / \mathrm{mL}$ ) and $P$. aeruginosa $10^{3} \mathrm{CFU} / \mathrm{mL}$. $P$. aeruginosa with the same antibiotic susceptibility pattern as previously was isolated and PCR for bla $a_{\text {NDM-1 }}$ was again positive. Kidney ultrasound was normal.

The patient received piperacillin-tazobactam (4g TID) for three weeks, and her second chemotherapy course, without complication. The urinalysis after 72 hours of treatment was negative and the patient was discharged for at-home hospitalisation.

During both hospitalisations, contact isolation precautions with dedicated healthcare personnel were implemented. All patients hospitalised in the same ward were screened weekly with a total of 111 rectal swabs performed in 52 patients and no transmission of NDM-1 producing $P$. aeruginosa occurred.

\section{Discussion and conclusion}

Since the first description in 2008, of an NDM-1 carbapenemase in single isolates of Klebsiella pneumoniae and Escherichia coli [3], NDM-1-producing Enterobacteriaceae have been reported worldwide, mostly in patients with an epidemiological link to India or Pakistan $[4,5]$. However, among 77 patients infected or colonised by NDM-1 producing Enterobacteriaceae reported in Europe from 2008 to 2010, five had been hospitalised previously in the Balkan region [6]. Clinical isolates of NDM-1-producing $A$. baumanii are also increasingly reported in Europe [7] and importation of NDM-1 -producing A. baumannii from Serbia has been reported in 2011 [8].

We report here the first case of infection due to NDM-1-producing $P$. aeruginosa in France. To date,

\section{TABLE 2}

Monitoring of plasmatic and urinary concentrations of antibiotics in patient with pyelonephritis caused by Pseudomonas aeruginosa serotype 011 (HIABP11) and history of previous hospitalisation in Serbia, France 2012

\begin{tabular}{|l|c|c|}
\hline & Aztreonam & Colistin \\
\hline Plasmatic peak concentration $(\mathrm{mg} / \mathrm{L})$ & 127.6 & NA \\
\hline Plasmatic residual concentration $(\mathrm{mg} / \mathrm{L})$ & 14.3 & 0.7 \\
\hline Urinary concentration $(\mathrm{mg} / \mathrm{L})$ & $>500$ & 22.3 \\
\hline
\end{tabular}

NA: not available. 
only two other cases of colonisation or infection by NDM-1-producing $P$. aeruginosa have been reported worldwide, occurring in two patients hospitalised in Belgrade,Serbia. Both had undergone invasive surgical interventions and none of them had travelled outside Serbia. No epidemiological connection was evidenced between them $[9,10]$.

NDM-1 producing bacteria are undoubtly challenging: firstly, they are usually multiresistant to antibiotics because bla $a_{\mathrm{NDM}-1}$ encoding plasmids co-harbor multiple resistance determinants. $P$. aeruginosa shows a high level of intrinsic resistance to antimicrobial agents. Its ability to acquire and combine different resistance determinants represents a major threat, compromising therapeutic options. The acquisition of MBL-carbapenemase (Verona integron-encoded metallo-beta-lactamase (VIM), imipenemase (IMP), Sao Paulo Metallo-beta-lactamase (SPM), Australia imipenemase (AIM), German imipenemase (GIM), Dutch IMipenemase (DIM), NDM, led to emergence of multidrug-resistant (MDR) or extensively drug-resistant (XDR) $P$. aeruginosa. The case presented highlights the difficulties of therapeutic management, with only three antibiotics categorised as susceptible or intermediate (colistin, aztreonam, piperacillin-tazobactam). Due to the low MIC recommended for the inferior breakpoint for aztreonam by EUCAST, wild type $P$. aeruginosa are reported as 'intermediate'. However MBLcarbapenemases do not hydrolyse the monobactam aztreonam and high dose therapy can be useful for patients infected with MBL-producing $P$. aeruginosa $[11,12]$.

Secondly, NDM-1 producers have a potential for spread through the transfer of the plasmid-borne $b a_{\mathrm{NDM}_{1}}$ gene [5]. In P. aeruginosa, there is no complete documentation for plasmid-borne or chromosomal localisation for bla $a_{\mathrm{NDM} 1}$ gene yet. However, many outbreaks including carbapenemase-producing $P$. aeruginosa and spread of MDR $P$. aeruginosa clones have been recently reported, underlining that cross-transmission plays a major role in the spread of MDR $P$. aeruginosa in hospital settings [13, 14]. These considerations combined with the emerging character of our isolate in France are reason why all members of the medical and paramedical staff agreed to set up a dedicated team to care for the patient and a weekly screening of all contemporary patients on the same ward.

This strategy is recommended in France for carbapenemase-producing Enterobacteriae [1] without any mention of carbapenemase-producing Acinetobacter or Pseudomonas. However, in this particular case of a first isolation of NDM-1 producing $P$. aeruginosa in France, this strategy allowed us to assess the absence of cross-transmission for this isolate.

This observation highlights the emergence of NDM-1 not only in Enterobacteriaceae, but also in $P$. aeruginosa in Balkan area and France. In our view, NDM screening should be performed when a carbapenemase-producing Pseudomonadaceae clinical isolate is identified.

\section{References}

1. Haut Conseil de la Santé Publique (HCSP). Maîtrise de la diffusion des bactéries multirésistantes aux antibiotiques importées en France par des patients rapatriés ou ayant des antécédents d'hospitalisation à l'étranger, zème version. [Control of the spread of multiresistant bacteria imported in France by repatriated patients or patients previously hospitalised abroad, 2nd version]. Paris: HCSP. Nov 2010. Available from: http://www.hcsp.fr/explore.cgi/ avisrapportsdomaine?clefr $=201$

2. European Committee on Antimicrobial Susceptibility Testing (EUCAST). EUCAST breakpoint tables for interpretation of MICs and zone diameters, Version 2.0] [Accessed $290 \mathrm{ct}$ 2012]. Available from: http://www.eucast.org/fileadmin/src/ media/PDFs/EUCAST files/Breakpoint tables/Breakpoint table_v_2.0_120221.pdf

3. Yong D, Toleman MA, Giske CG, Cho HS, Sundman K, Lee K, et al. Characterization of a new metallo-beta-lactamase gene, bla(NDM-1), and a novel erythromycin esterase gene carried on a unique genetic structure in Klebsiella pneumoniae sequence type 14 from India. Antimicrob Agents Chemother. 2009;53(12):5046-54.

4. Kumarasamy KK, Toleman MA, Walsh TR, Bagaria J, Butt F, Balakrishnan R, et al. Emergence of a new antibiotic resistance mechanism in India, Pakistan, and the UK: a molecular, biological, and epidemiological study. Lancet Infect Dis. 2010 Sep;10(9):597-602.

5. Nordmann P, Poirel L, Walsh TR, Livermore DM. The emerging NDM carbapenemases. Trends in microbiology. 2011;19(12):588-95.

6. Struelens MJ, Monnet DL, Magiorakos AP, Santos O'Connor F, Giesecke J, the European NDM-1 Survey Participants. New Delhi metallo-beta-lactamase 1-producing Enterobacteriaceae: emergence and response in Europe. Euro Surveill. 2010;15(46):pii=19716. Available from: http://www. eurosurveillance.org/ViewArticle.aspx?Articleld=19716

7. Bonnin RA, Poirel L, Naas T, Pirs M, Seme K, Schrenzel J, et al. Dissemination of New Delhi metallo-beta-lactamase-1producing Acinetobacter baumannii in Europe. Clin Microbiol Infect. 2012;18(9):E362-5.

8. Pfeifer Y, Wilharm G, Zander E, Wichelhaus TA, Gottig S, Hunfeld KP, et al. Molecular characterization of blaNDM-1 in an Acinetobacter baumannii strain isolated in Germany in 2007. J Antimicrob Chemother. 2011;66(9):1998-2001.

9. Livermore DM, Walsh TR, Toleman M, Woodford N. Balkan NDM-1: escape or transplant? Lancet Infect Dis. 2011;11(3):164.

10. 10.Jovcic B, Lepsanovic Z, Suljagic V, Rackov G, Begovic J, Topisirovic L, et al. Emergence of NDM-1 metallo-betalactamase in Pseudomonas aeruginosa clinical isolates from Serbia. Antimicrob Agents Chemother. 2011;55(8):3929-31.

11. Moriyama B, Henning SA, Childs R, Holland SM, Anderson VL, Morris JC, et al. High-dose continuous infusion betalactam antibiotics for the treatment of resistant Pseudomonas aeruginosa infections in immunocompromised patients. Ann Pharmacother. 2010;44(5):929-35.

12. Prevotat A, Leroy S, Perez T, Wallet F, Wallaert B. [Tolerance and efficacy of ceftazidime in combination with aztreonam for exacerbations of cystic fibrosis]. Rev Mal Respir. 2010;27(5):449-56.

13. Cholley $P$, Thouverez M, Hocquet D, van der Mee-Marquet $N$, Talon D, Bertrand X. Most multidrug-resistant Pseudomonas aeruginosa isolates from hospitals in eastern France belong to a few clonal types. J Clin Microbiol. 2011;49(7):2578-83.

14. Garcia-Castillo M, Del Campo R, Morosini MI, Riera E, Cabot G, Willems R, et al. Wide dispersion of ST175 clone despite high genetic diversity of carbapenem-nonsusceptible Pseudomonas aeruginosa clinical strains in 16 Spanish hospitals. J Clin Microbiol. 2011;49(8):2905-10. 